\title{
Nanoparticles as Antimicrobial Agents
}

\author{
Moharam Maysa* \\ Microbial Chemistry Department, Genetic Engineering and Biotechnology Division, National Research Centre Dokki, Giza, Egypt \\ *Corresponding author: Moharam Maysa, Microbial Chemistry Department, Genetic Engineering and Biotechnology \\ Division, National Research Centre Dokki, Giza, Egypt
}

\section{ARTICLE INFO}

Received: 慙 June 26, 2021

Published: 㓞 September 20, 2021

\section{ABSTRACT}

Citation: Moharam Maysa. Nanoparticles as Antimicrobial Agents. Biomed J Sci \& Tech Res 38(5)-2021. BJSTR. MS.ID.006217.

\section{Opinion}

Microbial infections are still a challenge despite of the existing of numerous potent antibiotic drugs and other modern antimicrobial means, Bacterial infections are still a major cause of mortality. The problem is that major groups of antibiotics, currently in use, generally affect three bacterial targets: cell wall synthesis, translational machinery, and DNA replication. Unfortunately, bacterial resistance may develop against each one of these modes of action. In addition, the use of conventional antibiotics carries a major risk for resistance of viable bacteria. Nanoparticles as antibacterial agents complementary to antibiotics are highly promising and are gaining large interest as they might fill the gaps where antibiotics frequently fail. This includes combatting multidrug-resistant mutants and biofilm. Nanotechnology is a technology conducted at the nano-scale in the fields of fabric manufacturing, food processing, agricultural processing, and engineering, as well as in medical and medicinal applications. Over the present decade, several studies have suggested that nanoparticles are excellent antibacterial agents, thus nanomaterial applications for antimicrobial works have prime interest by many researchers. Several reports showed that some of the metal oxide nanoparticles have toxicity toward several microorganisms and they could successfully kill numerous bacteria. This new approach has been identified to deal with resistance of pathogenic microorganisms because of their inherent antimicrobial activity. The use of nanoparticles as antimicrobial components especially in the food additives and medical applications can be one of the new and considerable strategies for overcoming pathogenic microorganisms. Based on literature review there are some effective factors that can influence the ability of nano materials in reducing or killing the cells. Mechanisms for nanomaterial against bacteria, which are briefly listed as follows: surface charge of the metal nanomaterial, shape, type and material, concentration of nanomaterial, dispersion and contact of nanomaterial to the bacterial cell, presence of active oxygen, liberation of antimicrobial ions, medium components and $\mathrm{pH}$, physicochemical properties, specific surface-area-to-volume ratios, size, role of growth rate, role of biofilm formation, and cell wall of bacteria.

Nanoparticles as antimicrobial agents have become an emerging approach which can establish an effective nanostructure to deliver the antimicrobial agents for targeting the bacterial community efficiently. In addition, they are so potent that microbial pathogens cannot develop resistance towards them. On the other hand, most of the metal oxide nanoparticles have no toxicity toward humans at effective concentrations used to kill bacterial cells, which thus becomes an advantage for using them in a full scale. Metal, metal oxide and organic nanoparticles now are used show a diversity of intrinsic and modified chemical composition properties. Thus, it is not surprising that they have numerous modes of action as antimicrobial agents. In some cases the ratio between the bacteria and the nanoparticles is critical to the bacterial toxicity. In addition, many factors play a role and affect the lethal effect of nanoparticles to bacteria including aeration, $\mathrm{pH}$, and temperature, size, shape, chemical modification and coating, and mixture in various ratios with other nanoparticles and solvent used. Lethal effect of nanoparticles is generally due to membrane damage occurs when nanoparticles bind electrostatically to the bacterial cell wall and membranes, leading to alteration of membrane 
potential, membrane depolarization, and loss of integrity which, in turn, result in an imbalance of transport, impaired respiration and cell death. Among inorganic metals silver nanoparticles have been widely used as an effective antimicrobial agent against bacteria, fungi, and viruses. The antimicrobial efficacy of silver (Ag), as of other metals and metal oxide nanoparticles, was reported to be size-dependent. Although the Ag nanoparticle mechanism of action is still not clear, small diameter Ag nanoparticles have a superior antimicrobial effect to those of a larger diameter In comparison to silver, gold- ( $\mathrm{Au}$-) nanoparticles are less potent and have almost no antibacterial effect by themselves.

Except when they of an antibacterial approach, adopted from cancer treatments, gold nanoparticles bound to Fe304 and activated by photothermal means. In addition, Titanium oxide with its photocatalytic effect serves as an antimicrobial agent for

\section{ISSN: 2574-1241}

DOI: $10.26717 /$ BJSTR.2021.38.006217

Moharam Maysa. Biomed J Sci \& Tech Res

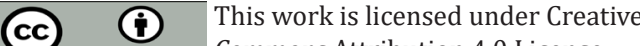
Commons Attribution 4.0 License

Submission Link: https://biomedres.us/submit-manuscript.php both positive and negative bacteria. An interesting approach in the antimicrobial application of nano- metals and nano- metal oxides is the synergistic effect of the combination between two or more of them as Titanium and silver. Moreover, Zinc oxide nanoparticles were shown to have a wide range of antimicrobial activity. This metal oxide is characterized by its low cost and low toxicity to human cells, thus it was used as coating materials designated for medical and other devices. White color, UV-blocking, and ability to prevent biofilm formation made its nano particles suitable for fabric treatment. Zinc was approved by the FDA as a food additive. Another metal oxide used as an antibacterial agent is copper oxide nanoparticles have been shown to be effective against various bacterial pathogens especially gram positive Bacilli. In our lab we obtained excellent results by applying these approaches in many fields of polymers and textile.

$\begin{array}{ll}\text { BIOMEDICAL } & \text { Assets of Publishing with us } \\ \text { RESEARCHES } & \text { - Global archiving of articles } \\ \text { - Immediate, unrestricted online access }\end{array}$

